\title{
DANIEL KAHNEMAN: A ECONOMIA MENTAL E O NOBEL DA ECONOMIA
}

\author{
Leonel Garcia-Marques e Mário A. B. Ferreira
}

Resumo Em Outubro de 2002 Daniel Kahneman foi galardoado pela Royal Swedish Academy of Sciences com o prémio Nobel da economia por ter integrado insights resultantes da investigação psicológica nas ciências económicas, especialmente no que concerne o julgamento humano e a tomada de decisão em condições de incerteza. Em Abril de 2003, os "Encontros ao Fim da Tarde com a Psicologia", da responsabilidade da Associação Portuguesa de Psicologia (APP) em articulação com a Livraria Barata, prestaram homenagem ao trabalho de Kahneman, apresentando, em duas sessões de divulgação, algumas das maiores contribuições deste autor para o estudo da decisão e julgamento na incerteza, e discutindo as consequências destas contribuições para a reflexão contemporânea sobre o conceito de racionalidade. Este artigo resume e discute as principais ideias apresentadas nestas sessões. ${ }^{1}$

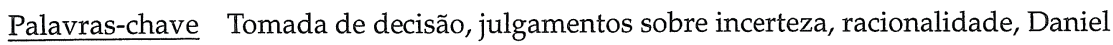
Kahneman.

\section{Introdução}

Em Outubro de 2002 a Royal Swedish Academy of Sciences atribuiu a Daniel Kahneman o prémio Nobel da economia pelo seu trabalho pioneiro sobre julgamento e tomada de decisão em condições de incerteza. Kahneman considerou que este prémio traduzia o reconhecimento público do seu trabalho teórico e de investigação de mais de 30 anos, desenvolvido em estreita colaboração com o seu amigo e colega Amos Tversky (falecido em 1996). O Nobel teria certamente sido partilhado pelos dois não fosse o facto de a Academia Sueca das Ciências não atribuir o prémio postumamente.

Este artigo, baseado numa sessão dos "Encontros ao fim da tarde com a Psicologia" organizados pela APP na Livraria Barata, visa prestar homenagem ao trabalho de Daniel Kahneman e Amos Tversky, apresentando de forma sucinta algumas das maiores contribuições dos autores para o estudo da decisão e julgamento em contextos de incerteza e discutindo as consequências dessas contribuições para a reflexão contemporânea sobre os conceitos de racionalidade económica e racionalidade adaptativa.

Na primeira parte, o conceito de heurísticas é apresentado como alternativa aos modelos racionais de escolha, no âmbito do programa de investigação em heurísticas e enviesamentos iniciado por Kahneman e Tversky nos início dos anos 70 (Tversky \& Kahneman, 1971; 1974). Duas das heurísticas, a heurística da representatividade e a heurística da disponibilidade, são sucintamente discutidas enquanto descrições dos processos cognitivos subjacentes ao raciocínio inferencial humano.

Leonel Garcia-Marques, Faculdade de Psicologia e de Ciências da Educação, Universidade de Lisboa; Mário A. B. Ferreira, Faculdade de Psicologia e de Ciências da Educação, Universidade de Lisboa. 
Na segunda parte discute-se o impacto que a proposta de heurísticas (não normativas) teve na economia. Com efeito, as heurísticas enquanto processos de julgamento e tomada de decisão colocam em causa a racionalidade do chamado homem económico, um conceito essencial para a economia moderna. Por outro lado, reformulam os princípios subjacentes às tomadas de decisão inteligentes eadaptativas, embora não necessariamente válidas ou racionais.

\section{Mais um Nobel da economia?}

A atribuição de mais um Nobel da economia nada teria de extraordinário, não fosse o caso de Kahneman ser um psicólogo, doutorado em psicologia em 1961 pela Universidade da Califórnia, enão um economista. De resto, o impacto nas ciências económicas da investigação psicológica que procura esclarecer os processos cognitivos subjacentes ao julgamento inferencial humano tem sido tal que Kahneman e Tversky são hoje considerados os fundadores duma disciplina das ciências económicas normalmente chamada economia comportamental (behavioral economics). Isto é tanto mais interessante quanto estes autores nunca tiveram qualquer intenção ou plano de forjar um novo campo de investigação em economia.

\section{O programa de investigação em heurísticas e enviesamentos}

Curiosamente, o argumento central do programa de investigação iniciado nos anos 70 por Kahneman e Tversky (1972; Tversky \& Kahneman, 1971; 1974) é muito simples: o julgamento inferencial humano afasta-se sistematicamente do prescrito pelos modelos racionais de escolha (e.g., teoria das probabilidades e estatística). Argumentos semelhantes já tinham sido anteriormente oferecidos por outros. Designadamente, ao propor a noção de racionalidade limitada, Simon (1957) tinha já chamado a atenção para os limites humanos no tratamento da informação disponível para tomar decisões. Por outro lado, se a investigação em julgamento e tomada de decisão descreveu inicialmente o homem como um estatístico intuitivo (Peterson \& Beach, 1967), a posterior acumulação de evidência experimental contrária mostrou claramente que os modelos racionais de escolha e tomada de decisão são maus descritores do desempenho inferencial humano (e.g., Sherman \& Corty, 1984). A proposta de Kahneman e Tversky introduz, no entanto, um corte radical com a teorização e investigação prévia (para revisões de literatura ver: Kahneman, Slovic \& Tversky, 1982; Gilovich, Griffin \& Kahneman, 2002). Estes autores defendem que o julgamento em condições de incerteza se baseia num conjunto limitado de heurísticas. Ou seja, processos simplificados de tomar decisões qualitativamente diferentes de quaisquer algoritmos de decisão explicitamente usados pelas pessoas ou empresas. As heurísticas envolvem basicamente a substituição automática de julgamentos de probabilidade por outros julgamentos mais fáceis. Neste sentido funcionam como uma espécie de "atalhos cognitivos" que nos permitem fazer escolhas e tomar decisões que envolvem incerteza de forma rápida e sem esforço. Mas vejamos alguns exemplos. 


\section{A heurística da representatividade}

Tversky e Kahneman (1983) apresentaram aos participantes de uma das suas experiências o problema da Linda. Imagine-se na pele destes participantes e leia atentamente:

A Linda tem 31 anos, é solteira, uma pessoa franca, e muito brilhante. Licenciou-se em filosofia. Enquanto estudante ela era profundamente interessada por questões de discriminação social e outros assuntos sociais, e participou em manifestações antinuclear.

Qual das seguintes alternativas é mais provável?

A Linda é bancária

A Linda é bancária e feminista

Se lhe parece que a alternativa b) é a mais provável, então, provavelmente, chegou a esta conclusão através daquilo a que Kahneman e Tversky chamaram a heurística da representatividade (Kahneman \& Tversky, 1972). ${ }^{2}$ De acordo com a heurística da representatividade, a probabilidade de um objecto A pertencer a uma categoria B ou de um acontecimento A ter origem num processo B é função da semelhança entre A e B. Ou seja, a probabilidade de um objecto pertencer a uma categoria está dependente do grau em que este objecto representa as características essenciais desta categoria.

Muitas vezes probabilidade e semelhança estão positivamente correlacionados. Neste casos, substituir um julgamento de probabilidade por um julgamento de semelhança não só permite realizar julgamentos rápidos e sem esforço mas sobretudo permite realizar julgamentos genericamente correctos ou satisfatórios. Infelizmente, os julgamentos por semelhança são insensíveis a diversas variáveis que afectam o cálculo de probabilidades. De facto, no caso do problema da Linda, a escolha da alternativa b) viola uma das regras de probabilidade mais básicas. A regra da conjunção de probabilidades: a probabilidade da conjunção de dois eventos é sempre igual ou inferior à probabilidade de ocorrência de apenas um destes eventos. Por outras palavras, a probabilidade de a Linda ser bancária e feminista é sempre inferior ou quanto muito igual à probabilidade de a Linda ser bancária. Com efeito, se pensarmos no conjunto de todas as mulheres que correspondem à descrição da Linda, a quantidade destas mulheres que são bancárias é necessariamente superior ou quanto muito igual ao número de mulheres que, para além de serem bancárias, são também feministas. Curiosamente, mesmo depois de reconhecer a lógica da regra da conjunção, muitos participantes reportam que a alternativa que descreve a Linda como bancária e feminista continua a ser intuitivamente mais crível. ${ }^{3}$

\section{A heurística da disponibilidade}

Por outro lado, muitas vezes julgamos a probabilidade de ocorrência de um dado evento através da facilidade com que recuperamos instâncias desse evento. A esta forma de julgamento na incerteza Kahneman e Tversky chamaram a heurística da disponibilidade (Tversky \& Kahneman, 1973). Por exemplo, Tversky e Kahneman (1973) verificaram que as pessoas em geral consideram que o número de grupos de dois elementos que podem ser gerados a partir de uma amostra de 10 elementos é superior ao número de grupos de oito elementos que podem ser gerados a partir da mesma amostra de 10 elementos. No entanto, o número de combinações possíveis de 10 elementos 2 a 2 é igual ao número de combinações de 10 elementos 8 a 8 . O cálculo deste número de combinações pode ser obtido com algum esforço, compilando todos os casos possíveis e contando-os, ou aplicando o algoritmo matemático adequado. ${ }^{4}$ Este cálculo pode, contudo, ser substituído sem esforço e quase imediatamente pela facilidade com que 
conseguimos trazer à memória casos específicos de dois e de oito elementos a partir de uma amostra de 10. É muito mais fácil e rápido gerar uma amostra mental de instâncias com um pequeno número de elementos do que instâncias com um grande números de elementos. Consequentemente, os participantes nesta experiência de Kahneman (Tversky \& Kahneman, 1973) julgaram erradamente os grupos de dois elementos como sendo muito mais frequentes ou prováveis do que os grupos de oito.

Para melhor ilustrar o funcionamento desta heurística imagine que lhe é pedido para estimar o número de palavras do português que acabam em "ente". Assim como, o número de palavras portuguesas cuja a antepenúltima letra é um " $n$ ". Intuitivamente, parece muito mais fácil trazer à memória palavras de acordo com o primeiro pedido do que de acordo com o segundo. Uma vez que quanto mais fácil for a recuperação desta informação em memória maiores são as estimativas subjectivas de palavras, tendemos a realizar estimativas maiores no primeiro, do que no segundo caso. Analogamente, estimativas realizadas por grupos independentes de sujeitos são mais altas para palavras em inglês com terminação "ing" do que para palavras cuja penúltima letra é um " $n$ " (Tversky \& Kahneman, 1983). No entanto, o número de palavras que satisfazem o primeiro pedido é um subcorjunto do número de palavras que estão de acordo com segundo pedido.

Em suma, a heurística da disponibilidade leva assim a respostas satisfatórias sempre que a amostra de instâncias recuperadas da memória seja representativa da população. No entanto, a nossa memória é afectada por diversos factores que enviesam a realização destas amostras mentais (e.g., efeitos de primazia e de recência, fenómenos de inibição retroactiva ou proactiva, etc.). ${ }^{5}$

Heurísticas enquanto ilusões cognitivas

Note-se que a estratégia de investigação de Kahneman e Tversky foi justamente a procura de condições experimentais que permitissem distinguir respostas de acordo com algoritmos formais de probabilidade de respostas baseadas em princípios heurísticos. Os resultados encontrados fundamentam empiricamente o modelo de julgamento heurístico proposto pelos autores. Neste sentido, a identificação dos erros e enviesamentos ${ }^{6}$ cometidos pelos participantes das engenhosas experiências de Kahneman e Tversky não são importantes per se mas porque possibilitaram a investigação dos processos cognitivos subjacentes ao raciocínio inferencial humano, exactamente como o estudo das ilusões perceptivas permitiu tirar ilações sobre o funcionamento do sistema perceptivo humano. Com efeito, numa clara alusão a esta analogia, Kahneman e Tversky consideraram que as heuristicas não são estratégias deliberadas de tomada de decisão mas antes avaliações naturais evocadas pelos próprios dados dos problemas que produzem frequentemente ilusöes cognitivas (Tversky \& Kahneman, 1974).

\section{O essencial do julgamento heurístico}

Em suma, a mensagem central do programa de investigação em heurísticas e enviesamentos iniciado por Kahneman e Tversky é a seguinte: o julgamento em condições de incerteza baseia-se num conjunto limitado de princípios heurísticos qualitativamente diferentes de quaisquer algoritmos ou modelos racionais de escolha explicitamente usados para o cálculo de probabilidades. As heurísticas, por sua vez, baseiam-se na sụbstituição automática de julgamentos de probabilidade por outros julgamentos mais fáceis, são em grande medida evocadas pelos dados do problema, e não envolvem esforço cognitivo ou intenção consciente.

O trabalho teórico e de investigação de Daniel Kahneman levanta sérios desafios aos modelos 
racionais de escolha que subjazem à grande maioria das teorias económicas. Este ponto será de resto discutido em mais promenor nas páginas seguintes. Mas talvez o mais importante a reter, até agora, é que esta impressionante contribuição para o desenvolvimento das ciências económicas foi feita por um psicólogo, professor de psicologia, que desde 1993 lecciona a famosa disciplina de introdução à psicologia (mais conhecida por "Psych 101") na Universidade de Princenton, nos EUA.

\section{O fim do mito da racionalidade económica}

A proposta de que heurísticas não normativas estariam subjacentes ao julgamento e decisão na incerteza criou ondas de choque em várias disciplinas. Uma dessas disciplinas foi a economia. E porquê? Porque colocou radicalmente em causa o conceito da racionalidade dos agentes económicos, um conceito essencial da economia moderna.

\section{Racionalidade ideal e racionalidade económica: razões impraticáveis}

O conceito de racionalidade em economia é um conceito ideal. É como os conceitos de recta, plano ou acaso, que só são actualizáveis no infinito. Tomemos o conceito clássico de acaso. Todos concordaríamos que uma sequência em que todos os acontecimentos possíveis fossem equiprováveis seria aleatória. E se parece simples definir o que são resultados possíveis, tal definição rapidamente se torna impraticável. Pensemos em lançamentos de uma moeda, como poderemos saber se ela é "honesta"? Verificando a equiprobabilidade dos casos possíveis. Para que a moeda se possa considerar "honesta", tem que se registar um número aproximadamente igual de caras e coroas. Mas não só. Esses não são os únicos acontecimentos possíveis. Temos de registar a equiprobabilidade dos pares de lançamentos (existem quatro: cara/cara, cara/coroa, coroa/cara e coroa/coroa). E dos trios (existem oito: cara/cara/cara, cara/co$\mathrm{roa} / \mathrm{cara}$, cara/cara/coroa, cara/coroa/coroa, coroa/cara/coroa, coroa/coroa/cara, coroa/cara/cara e coroa/coroa/coroa). E assim sucessivamente... Daí que, de facto, só poderíamos considerar uma moeda como matematicamente "honesta" se dispuséssemos de um número infinito de observações. ${ }^{7}$ O conceito de acaso é, assim, um ideal, não uma categoria do nosso universo empírico.

O conceito de homem económico, em especial no que concerne o seu componente de racionalidade, é também um conceito ideal. Senão vejamos, segundo a concepção clássica de Von Neumann e Morgenstern (Edwards, 1954; Luce \& Raiffa, 1957), o homem económico possuiria as seguintes competências cognitivas em tomada de decisão na incerteza:

- conhece todos os cursos de acção possíveis e suas consequências prováveis;

- é capaz de atribuir probabilidades às possíveis consequências de cada curso de acção;

- é capaz de atribuir diferentes graus de preferência às possíveis consequências de cada curso de acção;

- a atribuição de probabilidades e a ordenação de preferências são independentes; é infinitamente discriminativo;

- é racional porque: (i) é capaz de ordenar as suas preferências; (ii) as suas escolhas possuem reflexividade, conectividade e transitividade; (iii) maximiza a utilidade esperada (subjectiva). 
Para apontar apenas um dos aspectos que torna este conjunto de competências impraticáveis basta tomar como exemplo a ordenação das preferências. Quão difícil será esta tarefa? As ciências da computação ajudam-nos a encontrar a resposta (Rich, 1986). Dentro dos problemas ditos resolúveis existe uma classe de problemas cuja resolução apesar de teoricamente possível se torna rapidamente impraticável, são os chamados problemas NP-completos (Cook, 1971). Tais problemas são considerados difíceis porque, à medida que os dados do problema crescem exponencialmente, o tempo necessário para um supercomputador conceptual o resolver aumenta também exponencialmente. Verificar a não-contradição de uma ordenação é precisamente um desses problemas. Senão vejamos, para verificar a consistência de uma ordenação temos de ser capazes de, dado um qualquer subconjunto dos objectos ordenados, encontrar uma sequência consistente com as posições que ocupam na ordenação geral. Como existem $2^{\mathrm{N}}$ subconjuntos possíveis de $\mathrm{N}$ objectos, à medida que $\mathrm{N}$ cresce exponencialmente o número de passos ou o tempo necessários para verificar a consistência dessa ordenação também cresce exponencialmente. Por exemplo, se um supercomputador virtual demorasse o tempo que um raio de luz demora a atravessar um protão, a encontrar a sequência consistente de cada subconjunto de objectos da ordenação, bastaria a ordenação conter mais de 138 objectos para o supercomputador demorar mais de vinte biliões de anos a realizar tal tarefa - o tempo que decorreu desde o Big Bang até aos nossos dias (Cherniak, 1986)...E Em problemas reais de decisão (ou jogos como o xadrez, o Go ou até o Tetris!) é necessário ordenar muito mais consequências do que as 138 citadas. Portanto, mesmo que fôssemos consistentes nas nossas preferências nunca poderíamos verificá-lo. A racionalidade económica é assim um conceito ideal tão longe do nosso mundo empírico como a imortalidade. Não é assim de espantar que a forma como tomamos decisões e realizamos julgamentos de probabilidades se afaste tanto da racionalidade virtual do homem económico.

Uma ressalva, tona-se, contudo, indispensável. É a de que a crítica da aplicação dos modelos normativos ideais ao mundo empírico não significa que esses modelos não continuem a ser importantes. Esses modelos normativos descrevem-nos os procedimentos formais que garantem a obtenção da melhor solução para os problemas de decisão, julgamento ou estimação. Mesmo se esses procedimentos forem impraticáveis no mundo empírico para problemas complexos, os modelos normativos continuam a ter uma função reguladora na identificação dos erros derivados do uso de heurísticas de decisão e julgamento ou na busca das melhores heurísticas.

A racionalidade heurística: transparência do contexto decisional e sensibilidade às consequências decisionais

Kahneman e Tversky deitaram por terra a esperança de descrever a actividade cognitiva humana a partir de modelos normativos ideais. Terão, por isso, Khaneman e Tversky sido arrastados (e nós com eles) para a posição niilista de que as nossas decisões ejulgamentos de probabilidades são absurdos, inúteis e ineducáveis? De forma nenhuma! Só é legítimo concluir é que as nossas decisões e julgamentos de probabilidades não são óptimos ou válidos a priori. É também legítimo pensar que podemos tornar essas decisões e julgamentos mais inteligentes, a partir de alguns princípios simples. Tomemos dois:

A transparência do contexto decisional. À medida que a investigação sobre julgamentos e decisões na incerteza avança, melhor identificamos as condições em que regras heurísticas de decisão e julgamento na incerteza produzem resultados convergentes com os da aplicação de modelos normativos e em que condições é que heurísticas e modelos normativos divergem (Garcia-Marques, Sherman, \& Oliveira, 2001; Kahneman \& Tversky, 1982b; McKenzie, 1992). Torna-se, por isso, extremamente importante 
garantir que as condições em que os decisores individuais operam sejam transparentes. Quer dizer, se aproximem daquelas em que heurísticas e modelos normativos convergem.

A sensibilidade às consequências decisionais. Muitas vezes, a racionalidade do processo de decisão encontra-se não no processo de escolha de uma linha de acção mas na forma como se modifica essa linha de acção em função dos resultados obtidos (Kahneman \& Tversky, 1982b). Hogarth (1981) demonstrou que um processo de decisão que se inicie com uma tomada de decisão aleatória se tende a aproximar de uma decisão válida desde que seja possível ao sistema decisor ir modificando as suas decisões, em função das suas consequências. Esta ideia está presente, por exemplo, nas redes neuronais ou modelos conexionistas de aprendizagem tutorada que funcionam segundo a regra delta de aprendizagem de Widrow e Hoff (Gluck \& Myers, 2001).

Estes princípios permitem, em princípio, que decisores heurísticos tomem decisões inteligentes e adaptativas, embora não necessariamente válidas ou racionais, no sentido ideal do termo, mas talvez heuristicamente racionais.

Caso contrário, poderemos sempre ignorar as contribuições de Kahneman e Tversky e fazer como Bertold Bretch recomendou num poema: "caso o povo critique o governo, demita-se o povo". Ou seja, caso os seres humanos se não ajustem aos modelos normativos, demitam-se os seres humanos.

\section{Notas}

1 Correspondência sobre este artigo deve ser dirigida para: Leonel Garcia-Marques, Faculdade de Psicologia e de Ciências da Educação, Alameda da Universidade, 1640-013 Lisboa, Portugal. E-mail: garcia_marques@sapo.pt

2 Inicialmente denominada "representation hypothesis" (Tversky \& Kahneman, 1971).

3 O paleontólogo americano Stephen G. Gould (1991) partilha desta intuição: "eu sei que a [conjunção] é menos provável, no entanto um pequeno homúnculo continua a saltar dentro da minha cabeça, gritando - mas ela não pode ser só uma bancária, lê a descrição [!]" (p. 469).

4 Combinações de $m$ elementos diferentes a partir de um conjunto $n$ podem ser obtidas através da fórmula: $\mathrm{n} ! / \mathrm{m} !(\mathrm{n}-\mathrm{m})$ !

5 Kahneman e Tversky propuseram ainda duas outras heurísticas. A heurística da ancoragem e ajustamento (Tversky \& Kahneman, 1974) e a heurística da simulação (Kahneman \& Tversky 1979; 1982A). A heurística da ancoragem e ajustamento estabelece que para muitos tipos de julgamentos as pessoas começam por realizar estimativas partindo de um valor inicial ou "âncora" e depois ajustam este valor até chegar ao julgamento final. Este ajustamento é, contudo, geralmente insuficiente. Por isto, os julgamentos finais são usualmente enviesados no sentido da "âncora" inicial. De acordo com a heurística da simulação, a probabilidade de ocorrência de um evento é função da facilidade de construção de cenários mentais hipotéticos onde o evento ocorre.

6 Definidos por comparação com modelos formais.

7 Os testes estatísticos de aleatoriedade não evitam este problema, apenas restringem para efeitos práticos a noção de casos possíveis e das suas iteracções. 


\section{Referências}

Cherniak, C. (1986). Minimal rationality. Cambridge, MA: Bradford Books, MIT Press.

Cook, S. (1971). The complexity of theorem-proving procedures". Proceedings of the $3^{\text {rd }}$ Annual ACM Symposium on Theory of Computing, 151-158.

Edwards, W. (1954). The theory of decision making. Psychological Bulletin, 51, 380-417.

Garcia-Marques, L., Sherman, S. J., \& Oliveira, J. M. P. (2001). Hypothesis-testing and perception of co-variation. Journal of Experimental Social Psychology, 37, 183-200.

Gilovich, T., Griffin, D., \& Kahneman, D. (2002). Heuristics and biases: The psychology of intuitive judgment. Cambridge: Cambridge University Press.

Gluck, M. A., \& Myers, C. E. (2001). Gateway to memory: An introduction to neural network modeling of the hippocampus and learning. Cambridge, MA: Bradford Books, MIT Press.

Gould, S. J. (1991). Bully for brontosaurus. Reflections in natural history. Nova Iorque: Norton.

Hogarth, R. M. (1981). Beyond discrete biases: Functional and dysfunctional aspects of judgmental heuristics. Psychological Bulletin, 90, 197-217.

Kahneman, D., \& Tversky, A. (1972). Subjective probability: A judgment of representativeness. Cognitive Psychology, 3, 430-454.

Kahneman, D., \& Tversky, A. (1979). Intuitive prediction: Biases and corrective procedures. TIMS Studies in Management Science, 12, 313-327.

Kahneman, D., \& Tversky, A. (1982a). The simulation heuristic. In D. Kahneman, P. Slovic, \& A. Tversky (Eds.), Judgment under uncertainty: Heuristics and biases (pp. 201-208). Cambridge: Cambridge University Press. .

Kahneman, D., \& Tversky, A. (1982b). Intuitive prediction: Biases and corrective procedures. In D. Kahneman, P. Slovic \& A. Tversky (Eds.), Judgment under uncertainty: Heuristics and biases (pp. 414-421). Cambridge: Cambridge University Press.

Kahneman, D., Slovic, P., \& Tversky, A. (Eds.) (1982). Judgment under uncertainty: Heuristics and biases. Nova Iorque: Cambridge University Press.

Luce, R. D., \& Raiffa, H. (1957). Games and decisions: Introduction and critical survey. Nova Iorque: Wiley and Sons.

McKenzie, C. R. M. (1992). The accuracy of intuitive judgment strategies: Co-variation assessment and bayesian inference. Cognitive Psychology, 26, 209-239.

Peterson, C. R., \& Beach. L. R (1967). Man as an intuitive statistician. Psychological Bulletin, 68, 29-46.

Rich, E. (1986). Artificial intelligence. Nova Iorque: McGraw-Hill.

Sherman, S. J., \& Corty, E. (1984). Cognitive heuristics. In R. S. Wyer \& T. K. Srull (Eds.), Handbook of social cognition (vol. 1, pp. 189-286.). Hillsdale, NJ: LEA.

Simon, H. (1957). Models of man: Social and rational. Nova Iorque: Wiley.

Tversky, A., \& Kahneman, D. (1971). Belief in the law of small numbers. Psychological Review, 2, 105-110.

Tversky, A., \& Kahneman, D. (1973). Availability: A heuristic for judging frequency and probability. Cognitive Psychology, 4, 207-232.

Tversky, A., \& Kahneman, D. (1974). Judgment under uncertainty: Heuristics and biases. Science, 185, 1124-1131.

Tversky, A., \& Kahneman, D. (1983). Extensional versus intuitive reasoning: The conjunction fallacy in probability judgment. Psychological Review, 90, 293-315. 
Daniel Kahneman: Mental economy and the Nobel of economy (abstract) In October 2002, Daniel Kahneman was ingratiated by the Royal Swedish Academy of Sciences with the Nobel prize of economy for his integration of insights that stemmed from psychological research on the economy field, especially in what concerns human judgement and decision making under uncertainty. In April 2003, the "Encontros ao Fim da Tarde com a Psicologia" organised by the Associação Portuguesa de Psicologia (APP), in colaboration with Livraria Barata, a tribute was payed to Kahneman's work, presenting, during two sessions, some of the greatest contributions of this author to the study of decision making and judgement under uncertainty, and discussing the consequences of these contributions to the contemporary thinking about the concept of rationality. The present paper summarises and discusses the main ideas presented during these sessions. 